most common malignancy in patients with Sjogren's syndrome was thyroid cancer. The risk of overall malignancy was not higher than that of the control. However, the incidence of prostate cancer (HR; 1.339, 95\% Cl; 1.076 1.667), thyroid cancer (HR; 1.320, 95\% Cl; 1.093 1.594) and lymphoma (HR; 1.620, 95\% Cl: 1.066 2.462) were higher and hepatocellular carcinoma (HR; $0.591,95 \% \mathrm{Cl}$; $0.455 \sim 0.767$ ) was lower than that of control. Lymphoma developed in 305 patients (3.1\% of total malignancy); 10 cases of Hodgkin lymphoma, and 290 cases of non-Hodgkin lymphoma. Gender, age, smoking, drinking, body mass index, fasting blood glucose, and proteinuria were not significantly different from the control group in lymphoma development.

Conclusions: Sjogren's syndrome patients' overall malignancy risk was not higher than that of control group. However, risk of lymphoma, prostate cancer, and thyroid cancer was higher than control group.

Disclosure of Interest: None declared

DOI: 10.1136/annrheumdis-2018-eular.2460

\section{SAT0415 THE MRZ REACTION HELPS TO DISTINGUISH RHEUMATOLOGIC DISORDERS WITH CENTRAL NERVOUS INVOLVEMENT FROM MULTIPLE SCLEROSIS}

N. Venhoff ${ }^{1}$, J. Thiel ${ }^{1}$, A.C. Venhoff ${ }^{1}$, S. Rauer ${ }^{2}$, D. Huzly ${ }^{3}$, M.-T. Schleyer ${ }^{1}$, C. Hentze ${ }^{1}$, R.E. Voll ${ }^{1}$, M. Rizzi ${ }^{1}$, U. Salzer ${ }^{1}$, T. Hottenrott ${ }^{2}$. ${ }^{1}$ Department of Rheumatology and Clinical Immunology, ${ }^{2}$ Department of Neurology and Neurophysiology; ${ }^{3}$ Institute of Virology, University Medical Center Freiburg, Freiburg, Germany

Background: Some rheumatologic disorders (RD) may initially manifest with central nervous system (CNS) affection, mimicking the clinical, magnetic resonance imaging, and cerebrospinal fluid (CSF) findings of multiple sclerosis (MS). Vice versa MS might be difficult to separate from some RD because of the presence of autoantibodies (e.g. ANA) in up to $50 \%$. The MRZ reaction (MRZR), composed of the three respective antibody indices (Al) against measles, rubella, and varicella zoster virus, has been found positive frequently in MS patients. However, it is unclear whether the MRZR is helpful to distinguish rheumatologic disorders with CNS involvement (RDwCNS) from MS.

Objectives: To investigate the MRZ reaction as a diagnostic tool to distinguish patients with RDwCNS from patients with MS.

Methods: The MRZR was evaluated in 35 patients with RDwCNS and compared to 70 sex- and age- matched MS patients. An Al result $\geq 1.5$ was indicative for intrathecal IgG production against the respective pathogen. Two previously established stringency levels, MRZR-1 ( $\geq 1$ of 3 Als positive) and MRZR-2 ( $\geq 2$ of 3 Als positive), were applied. CNS involvement of RDwCNS was defined as clinical manifestation with neurological symptoms and signs of inflammation in CSF analysis and/or cerebral/spinal magnetic resonance imaging (MRI). MRZR results were compared using the Fisher's exact test with $p<0.05$ for statistical significance.

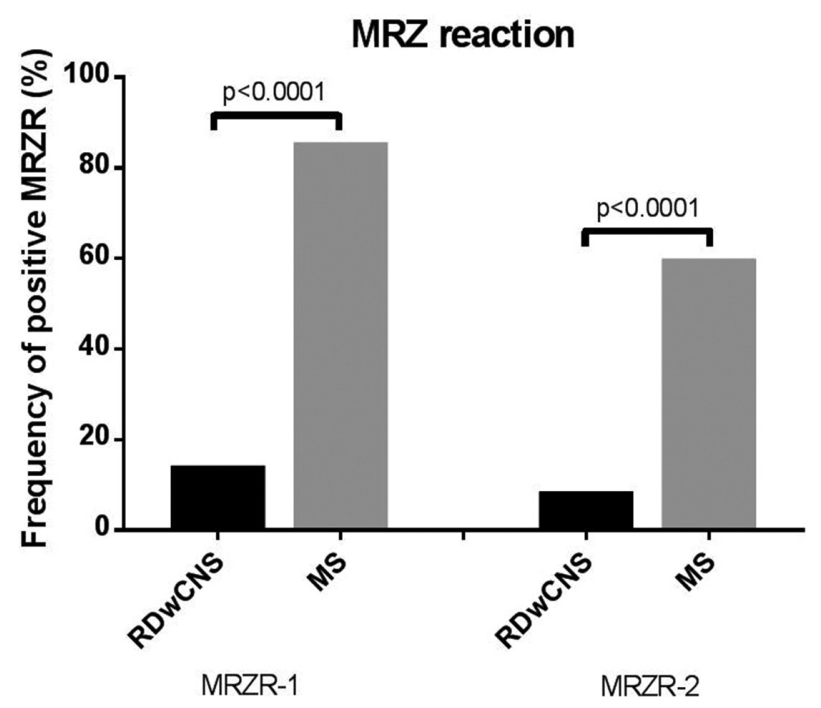

Abstract SAT0415 - Figure 1. Frequency of positive MRZ reaction (MRZR-1 and MRZR-2) in patients with RDwCNS compared to MS patients.

Results: Within the RDwCNS group, 31 patients suffered from systemic lupus erythematosus, four had a small vessel vasculitis. In both groups $77.1 \%$ were female, mean age $( \pm S D)$ was 43.2 years $( \pm 18.7)$ in RDwCNS and 47.5 years
$( \pm 7.8)$ in MS ( $p=n$.s.). All RDwCNS patients showed clinical symptoms indicative for CNS involvement and signs of inflammation in CSF analyses and/or MRI of the brain. In 52 MS patients autoantibody screening was performed. $42 \%$ were positive for ANA $(n=20)$ or ANCA $(n=2)$ in indirect immunofluorescence. Only $14.3 \%$ of RDwCNS patients had a positive MRZR-1 compared to $85.7 \%$ within the MS group $(p<0.0001)$. The more specific MRZR-2 was positive in $60 \%$ of the MS patients compared to only $8.5 \%$ of the RDwCNS patients $(p<0.0001)$. By using a higher threshold of $>2.0$ for a positive Al, the prevalence of positive MRZR-2 dropped to $5.7 \%(n=2)$ in the RDwCNS group compared to $54.3 \%(n=38)$ in the MS group $(p<0.0001)$. Oligoclonal bands were found in $94.3 \%$ of the MS and $28.6 \%$ of the RDwCNS patients $(p<0.0001)$.

Conclusions: Considering the high specificity of the MRZR-2 for MS confirmed in this study, this laboratory test may be a helpful diagnostic tool to distinguish RDwCNS from MS.

Disclosure of Interest: None declared

DOI: 10.1136/annrheumdis-2018-eular.3112

\section{SAT0416 LIFE-THREATENING PRIMARY SJÖGREN SYNDROME: CLINICAL CHARACTERISATION AND OUTCOMES IN 1535 PATIENTS (GEAS-SS REGISTRY)}

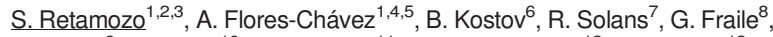
B. Maure ${ }^{9}$, C. Feijoo ${ }^{10}$, F.J. Rascón ${ }^{11}$, R. Pérez-Alvarez ${ }^{12}$, M. Zamora $^{13}$, A. GarcíaPérez ${ }^{14}$, M. Lopez-Dupla ${ }^{15}, M$. Á. Duarte Millán ${ }^{16}, M$. Ripoll ${ }^{17}$, E. Fonseca $^{18}$ P. Guisado-Vasco ${ }^{19}$, B. Pinilla ${ }^{20}$, G. de la Red ${ }^{21}, S$. Rodríguez Rodríguez ${ }^{22}, M$. J. Soto-Cárdenas ${ }^{23}$, P. Fanlo ${ }^{24}$, C. Morcillo ${ }^{25}$, M. Ramos-Casals ${ }^{1}$, P. BritoZerón ${ }^{1,25}$ on behalf of the GEAS-SS SEMI Registry. ${ }^{1}$ Hospital Clinic, IDIBAPS, Barcelona, Spain; ${ }^{2}$ Hospital Privado Universitario de Cordoba, IUCBC; ${ }^{3}$ INICSA, UNC-CONICET, Cordoba, Argentina; ${ }^{4} \mathrm{CMNO}$, IMSS, Guadalajara; ${ }^{5} \mathrm{CUIB}$, Universidad de Colima, Colima, Mexico; ${ }^{6}$ Transverse Group for Research in Primary Care, IDIBAPS; ${ }^{7}$ Hospital Vall d'Hebron, Barcelona; ${ }^{8}$ Hospital Ramón y Cajal, Madrid; ${ }^{9}$ Complejo Hospitalario Universitario, Vigo; ${ }^{10}$ Hospital Parc Taulí, Sabadell; ${ }^{11}$ Hospital Son Espases, Palma de Mallorca; ${ }^{12} \mathrm{HAC}$, Vigo; ${ }^{13} \mathrm{Hospital}$ Virgen de las Nieves, Granada; ${ }^{14}$ Hospital Universitario Central de Asturias, Oviedo; ${ }^{15} \mathrm{Hospital}$ Joan XXIII, Tarragona; ${ }^{16} \mathrm{Hospital}$ de Fuenlabrada, Fuenlabrada; ${ }^{17}$ Hospital Infanta Sofía, Madrid; ${ }^{18}$ Hospital de Cabueñes, Gijón; ${ }^{19}$ Complejo Hospitalario Ruber Juan Bravo; ${ }^{20}$ Hospital Gregorio Marañón, Madrid;

${ }^{21}$ Hospital Esperit Sant, Santa Coloma de Gramenet, Barcelona; ${ }^{22}$ Hospital Universitario de Salamanca, Salamanca; ${ }^{23}$ University of Cadiz, Cadiz; ${ }^{24} \mathrm{Hospital}$ Virgen del Camino, Pamplona; ${ }^{25}$ Hospital CIMA-Sanitas, Barcelona, Spain

Objectives: To analyse the clinical features and outcomes of patients presenting with life-threatening systemic disease in a large cohort of Spanish patients with primary Sjogren syndrome (SjS)

Methods: The GEAS-SS multicenter registry was formed in 2005 with the aim of collecting a large series of Spanish patients with primary SS. By January 2018 the database included 1535 consecutive patients fulfilling the 2002/2016 criteria. Life-threatening systemic disease was defined as an activity level scored as "High" in at least one ESSDAI domain.

Results: 209 (14\%) were classified as presenting with a life-threatening systemic disease: 194 presented one ESSDAl domain classified as high, 14 two domains and only one presented three high activity domains. The high-ESSDAI domains included lymphadenopathy in $78(37 \%)$ cases, CNS in $28(13 \%)$, PNS in 25 (12\%), pulmonary in $25(12 \%)$, renal in $22(10 \%)$, cutaneous in $18(9 \%)$, articular in $18(9 \%)$, haematological in $7(3 \%)$ and muscular in $4(2 \%)$; the most frequent clinical presentations in each domain were, respectively, parotid lymphoma $(n=41)$, focal neurological deficit $(n=20)$, ganglionopathy $(n=11)$, usual interstitial pneumonitis $(n=9)$, renal failure $(n=11)$, ulcerated cutaneous vasculitis $(n=9)$, symmetric polyarthritis $(m=17)$, severe thrombocytopenia $(n=3)$ and severe myositis $(n=3)$. With respect to therapeutic approach, $144(69 \%)$ required glucocorticoids, $65(31 \%)$ immunosuppressive agents and $42(20 \%)$ biological therapies. During the follow-up, $36(17 \%)$ patients died, mainly due to lymphoma $(n=16)$, pulmonary fibrosis $(n=5)$, end-stage renal failure $(n=4)$, CNS progressive disease $(n=3)$ and systemic vasculitis $(n=3)$.

Conclusions: A $14 \%$ of patients with primary SjS develop a potentially life-threatening systemic disease (mainly lymphoma, but also severe internal organ involvements including nervous system, the lungs and the kidneys). This subset of patients requires intensive therapeutic management with a mortality rate of nearly $20 \%$ of cases.

Disclosure of Interest: None declared

DOI: 10.1136/annrheumdis-2018-eular.5114 